\title{
Thermopower of Nanocarbon Materials with Different Structure and Phase Composition
}

\author{
Iryna Ovsienko ${ }^{1}$, Lyudmyla Matzui ${ }^{1}$, Iryna Pundyk ${ }^{1}$, Yuriy Prylutskyy ${ }^{2} \&$ Yuri M. Strzhemechny ${ }^{3}$ \\ ${ }^{1}$ Deptartment of Physics, Kyiv National Taras Shevchenko University, Kyiv, Ukraine \\ ${ }^{2}$ Deptartment of Biophysics, Kyiv National Taras Shevchenko University, Kyiv, Ukraine \\ ${ }^{3}$ Department of Physics and Astronomy, Texas Christian University, Fort Worth, USA \\ Correspondence: Yuri M. Strzhemechny, Department of Physics and Astronomy, Texas Christian University, \\ TCU Box 298840, Fort Worth, TX 76129, USA. Tel: 1-817-257-5793. E-mail: Y.Strzhemechny@tcu.edu
}

Received: February 27, 2012 Accepted: March 15, $2012 \quad$ Published: May 24, 2012

doi:10.5539/jmsr.v1n3p19 URL: http://dx.doi.org/10.5539/jmsr.v1n3p19

The research is financed by STCU grant $N 4908$

\begin{abstract}
Experimental data are presented on the temperature dependence of thermopower of compacted specimens of nanocarbon materials (NCMs) with different structure and phase composition. The experiments revealed a substantial correlation between the structure/phase composition of NCMs and the thermopower mechanisms in these materials.
\end{abstract}

Keywords: nanocarbon, phase composition, thermopower

\section{Introduction}

It is known that nanocarbon materials (NCMs) could contain ordered (single-wall and multi-wall carbon nanotubes (CNTs) as well as nano-graphite particles) and disordered (amorphous nanocarbon particles, nano-onions, different fractal structures etc.) carbon phases alongside with the particles of a metal catalyst. Elucidation of the transport properties in such materials is a rather complicated problem. Each phase is characterized by a certain conductivity mechanism. Thus, the conductivity of a nano-graphite is described by the Klein model of 2D-graphite electronic structure. Within this model, the valence and conduction bands are assumed to be linked at the vertices of the Brillouin zone and the Fermi level is shifted into the valence band (Klein, 1964). This model is also used to describe conductivity of multi-wall CNTs (Song et al., 1994). Some authors suggest to use the model of hopping conductivity with variable hopping lengths (the so-called strong localization in the systems of a semiconducting nature in the presence of local disorder) to describe the transport properties of multi-wall CNTs (Shiraishi et al., 2002; Liu et al., 2001). This model is used to describe kinetic properties of amorphous carbon (Vovchenko et al., 2000). However, no systematic data on the NCM thermopower are available. This work presents studies of thermopower in compacted NCMs with different structural and phase composition.

\section{Experimental}

The thermopower measurements were performed for three batches of samples. Batch \#1 consisted of compacted NCM specimens containing CNT with a low number of walls without any binder. This NCM was synthesized by a catalytic deposition of a carbon-containing gas (CO) using the Ni/Y catalyst (Kang et al., 2008; Ovsienko et al., 2007). The specimens in batches \#2 and \#3 have been produced by a low-temperature catalytic decomposition of carbon monoxide, with iron oxide (batch \#2) and cobalt oxide (batch \#3) used as catalysts (Prilutskiy et al., 2005). Subsequently, the source NCM was processed by a thermo-chemical treatment to remove amorphous carbon particles and catalyst refuses (Ovsienko et al., 2006). The treatment sequence was as follows: annealing at $550^{\circ} \mathrm{C}$ for $30 \mathrm{~min}$ in air and then washing the specimens in a hydrochloric acid solution. Detailed analysis of the NCM structure and phase composition performed in (Ovsienko et al., 2006; Ovsienko et al., 2008) by the $\mathrm{X}$-ray diffraction (XRD) and electron microscopy showed that NCM of batch \#2 contains tube-like formations $70-300 \mathrm{~nm}$ in diameter and disordered carbon phase particles $\sim 100-300 \mathrm{~nm}$ in size. The XRD patterns for 
these NCMs contained only the (002) reflection of graphite. This reflection corresponds to the diffraction of the ordered graphite phase (nano-graphite and multi-wall CNTs with the interplanar distance of $0.335 \mathrm{~nm}$ ) and the low-order carbon phase (amorphous carbon with $d_{002}=0.342 \mathrm{~nm}$ ). The specimens in batch $\# 3$ contained only the ordered carbon phase $\left(d_{002}=0.335 \mathrm{~nm}\right)$ in a form of tube-like formations $\sim 200-500 \mathrm{~nm}$ in diameter and the spheroids made of fiber-like structures $\sim 50-70 \mathrm{~nm}$ in diameter.

These NCMs samples were compacted by cold pressing with $20 \%$ mass of polyvinyl acetate using the procedure described in (Ovsienko et al., 2007).

The thermoelectric power of the obtained specimens was studied in the 4.2-293 $\mathrm{K}$ temperature range employing the technique described in (Vovchenko et al., 2000; Matzui et al., 2005).

\section{Results and Discussion}

Figure 1 presents the temperature dependence of the thermoelectric power, $S(T)$, for NCM \#1. One can clearly see an approximately linear increase of S with temperature. It is well known that the multi-wall CNT band structure is represented by two touching cones in the Brillouin zone vertices (Ovchinnikov et al., 1998). Herewith, the Fermi level is slightly shifted into the valence band. A similar type of a band structure is exhibited, for example, by a turbostratic graphite (Vovchenko et al., 2000). A turbostratic graphite with the crystallite size of $\sim 20 \mathrm{~nm}$ is a disordered structure with no dominant crystallite orientation. Figure 1 also shows the temperature dependence of the thermopower in the turbostratic graphite, which is approximately linear as well. As was shown in (Ovsienko et al., 2001), the positive slope is due to the higher concentration of holes than electrons in this material. According to Figure 1, the $S(T)$ dependence for specimen \#1 is similar to that of a turbostratic graphite. The linear dependence on temperature is typical for the diffusion thermopower of a singular state of charge carriers with one carrier type (Piraux, et al., 1988)

$$
S_{d}=\frac{\pi^{2} k_{B}^{2} T}{3 e E_{F}}(1+p),
$$

where $k_{b}$ is the Boltzmann constant, $E_{F}$ is the Fermi energy, $p$ is a parameter determined by the prevailing type of charge carrier scattering. From equation (1), using $p=-0.5$ (scattering on crystallite surfaces) it is possible to estimate the value of the Fermi energy, $\sim 0.15 \mathrm{eV}$.

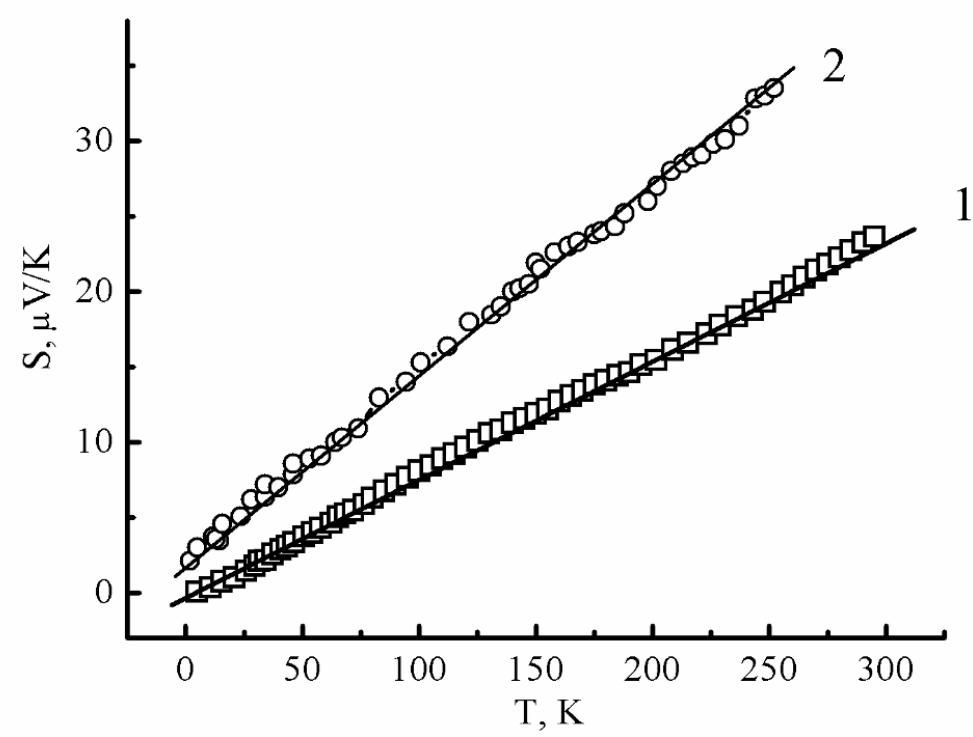

Figure 1. $S(T)$ dependence for: 1 - NCM of batch \#1;2 - turbostratic graphite

Figure 2 presents the $S(T)$ dependence for the batch \#2 specimens containing tube-like formations and particles of a disordered quasi-amorphous carbon phase. For comparison, Figure 2 also presents the temperature dependencies of the thermopower for quasi-amorphous carbon specimens. 


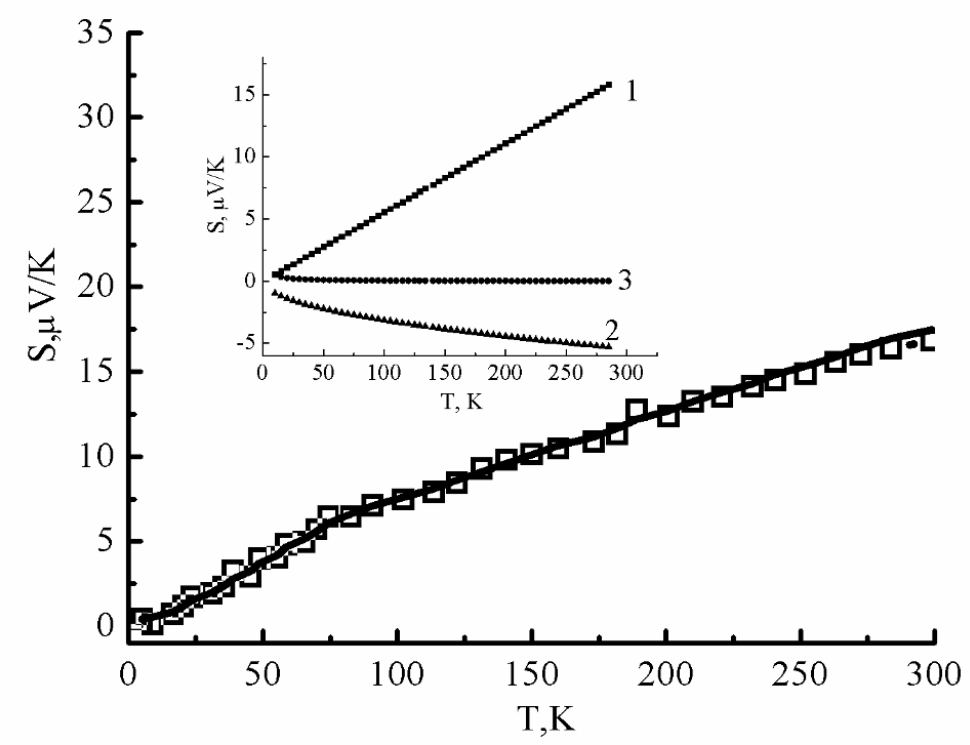

Figure 2. $S(T)$ dependence for NCM of batch \#2: circles represent experiment; solid curve represents calculation (Eq. 2-5); insert represents calculation of thermopower corresponding to the different types of conductivity: 1 -

$$
S_{1} ; 2-S_{2} ; 3-S_{3} \text { (see text) }
$$

A minimum is observed in the $S(T)$ dependence within the 20 - $30 \mathrm{~K}$ temperature range followed by a monotonic increase for specimen $\# 2$ as well as for other quasi-amorphous graphite materials. According to the reference data (Ovsienko et al., 2006), such structures could be considered as hetero-phase structures. Several conductivity mechanisms could be realized in these systems due to the presence of carbon phases with different levels of ordering (Hishiyama et al., 1978; Kaiser et al., 2001). If a certain phase in the studied specimens does not percolate, then the total conductivity is determined as the conductivity of the serially connected segments of a phase with a different conductivity type. According to this model the total thermopower $S$ of the carbon material could be expressed (Vovchenko et al., 2000) as a sum of thermopowers corresponding to the different conductivity types occurring in the material

$$
S=S_{1}+S_{2}+S_{3}
$$

where $S_{1}$ corresponds to the metallic conductivity phase, $S_{2}$ - to the hopping conductivity phase with variable hopping lengths, and $S_{3}$ - to the hopping conductivity phase with a constant hopping length. Thermoelectric power $S_{1}$ in the regions with metallic conductivity is described by Eq. (1). The thermoelectric power in the hopping conductivity regions with variable hopping lengths can be presented as (Ovsienko et al., 2007)

$$
S_{2}=\left.\frac{k_{b}^{2}}{2 e} \cdot\left(T_{0} \cdot T\right)^{1 / 2} \cdot \frac{d \ln N(E)}{d E}\right|_{E=E_{F}},
$$

where $N\left(E_{F}\right)$ is the density of electronic states at the Fermi level and $T_{0}$ is a constant that can be determined from the resistivity measurements. According to (Kotosonov, 1979), a linear correlation between the energy and density of electronic states near the joint point of the valence and conductivity bands should be realized

$$
N(E)=B|E|
$$

where $B$ is the appropriate coefficient and $E$ is the energy referred to the bands joint point. The sign of the $d N / d E$ derivative determines the sign of $S_{2}$. The thermoelectric power in the hopping conductivity regions with a constant hopping length can be described by the following expression (Vovchenko et al., 2000) 


$$
S_{3}=\frac{k_{b}}{2 e} \cdot\left(\frac{\varepsilon}{k_{b} T}+C\right), \varepsilon=E_{C}-E_{F}
$$

where $E_{c}$ is the mobility edge, $\varepsilon>0, C$ is a constant, which may be either negative or positive. From the experimental temperature dependence of the thermopower for sample \#2 we calculated the following parameters: the Fermi level shift in the regions with metallic conductivity $\Delta$, the Fermi energy in the regions with hopping conductivity of variable hopping length $E_{F}$, and the difference between the mobility edge and the Fermi level, $\varepsilon$. The results of calculations for these parameters, as well as the value of $T_{0}$, determined from the resistivity measurements, are given in Table 1. For comparison this table also contains the values of the relevant parameters for amorphous carbon (Vovchenko et al., 2000), in reasonable agreement with the ones calculated herein. The temperature dependence of the thermoelectric power for the studied specimens was calculated using the obtained values for the $\Delta, E_{F}$, and $\varepsilon$ parameters (Figure 2, insert). It was found that the calculated $S(T)$ dependence correlates well with the experimental behavior obtained at low temperatures.

Table 1. The results for calculations of parameters $T_{0}, E_{F}, \Delta, \varepsilon$

\begin{tabular}{lllll}
\hline Specimen & $T_{0}, \mathrm{~K}$ & $E_{F}, \mathrm{eV}$ & $\Delta, \mathrm{eV}$ & $\varepsilon, \mathrm{eV}$ \\
\hline NCM, Batch \#2 & 1139 & 0.4 & 0.20 & $3.5 \cdot 10^{-5}$ \\
Amorphous carbon (Vovchenko, et al., 2000) & 1967 & 0.4 & 0.45 & $4.4 \cdot 10^{-5}$ \\
\hline
\end{tabular}

The $S(T)$ dependence for specimen \#3 is presented in Figure 3. It differs substantially from that for others specimens. As was shown in (Ovsienko et al., 2006) and stated above, specimen \#3 contains only the ordered carbon phase with the interplanar distance of $0.336 \mathrm{~nm}$ similar to that in, e. g., fine crystalline graphite.

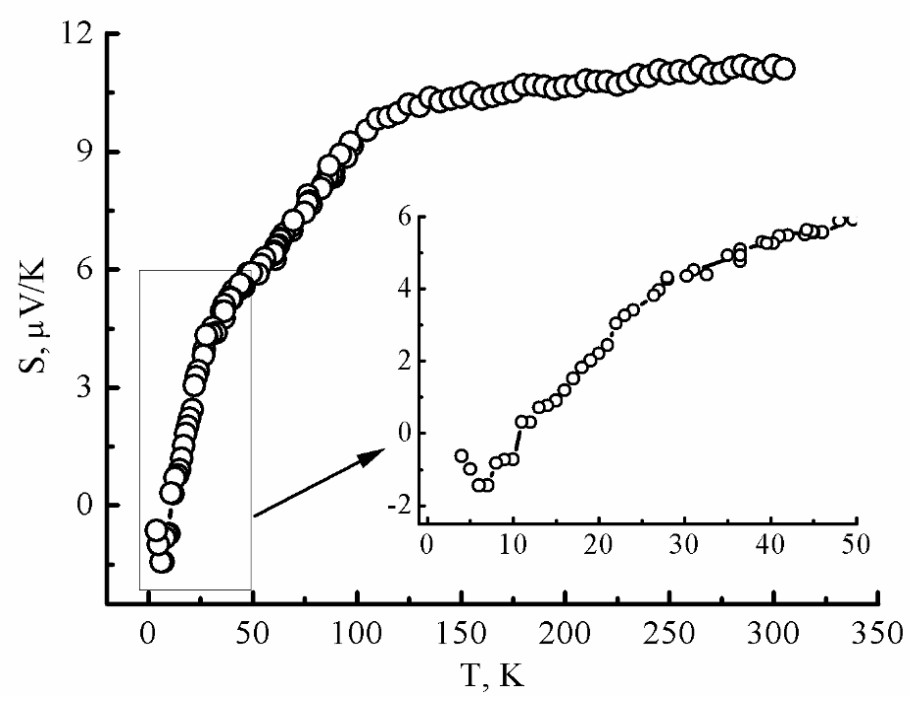

Figure 3. $S(T)$ dependence for NCM of batch \#3

For a more detailed analysis of the possible origins of thermopower in this sample further studies of the temperature dependence of its resistance were performed, the results of which are shown in Figure 4 . Figure 4 presents the temperature dependences of the resistivity $\left(R / R_{293}(T)\right)$ for all specimens. It clearly demonstrates that the $R_{4.2} / R_{293}$ ratio is equal to 1.3 for specimen \#3, that is typical for a fine crystalline graphite (Ovsienko et al., 2001). This is in contrast with specimens \#2 and \#1, for which this ratio is equal to 10 and 30 , respectively, typical for an amorphous carbon (Vovchenko et al., 2000). Thus, from the analysis of the structure, the phase composition and the transport properties of specimen \#3, one can assume that its thermopower can be described by a model that involves the diffusion component of a thermopower $S_{d}$ and a component associated with a 
phonon capture by the charge carriers, $S_{p h}$ (Ovsienko, et al., 2001):

$$
S=S_{d}+S_{p h}
$$

The contribution of the phonon component of the thermopower is significant at low temperatures when the phonon-phonon scattering is weak. When the temperature increases, the role of the phonon-phonon interaction increases and the magnitude of $S_{p h}$ tends to zero. Thus, at room temperature, the total thermopower is determined mainly by the contribution of the diffuse component of the thermopower.

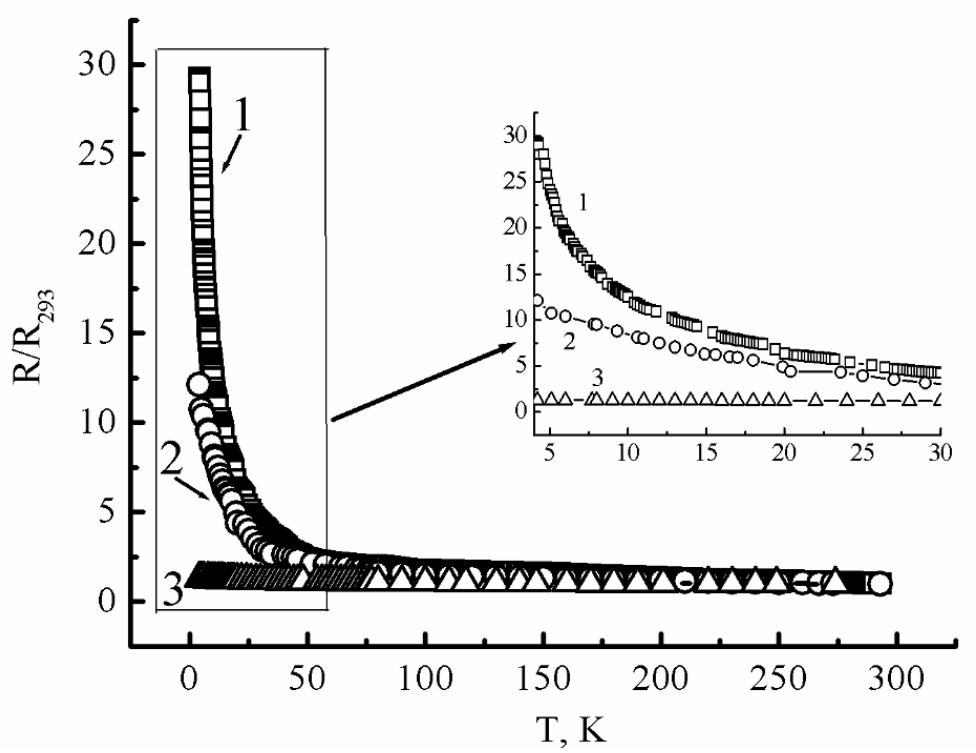

Figure 4. $R / R_{293}(T)$ dependence for NCMs: 1 - batch \#1, 2 - batch \#2, 3 - batch \#3

According to our estimation, the maximum of $S_{p h}$ occurs at a temperature below $10 \mathrm{~K}$ for NCM with the mentioned parameters of the crystal structure. This thermopower component itself does not exceed $2 \mu \mathrm{V} / \mathrm{K}$. The total thermopower temperature dependence is determined primarily by the diffusion contribution. Moreover, weak localization effects and the electron-electron interaction could influence the temperature dependencies of resistivity and thermopower for these materials in a presence of a weak structural disorder.

\section{Conclusion}

Studies of the temperature dependence of thermopower for different NCMs revealed a substantial correlation between the structure, phase composition and thermopower mechanisms in these nanomaterials. The thermopower of NCM containing CNTs with a low number of walls and a substantial amount of disordered carbon was shown to be dominated by the diffusion component that depends on the temperature linearly. The thermopower of NCM containing multi-wall CNTs with structural parameters close to those for amorphous carbon was shown to be described within the model of a serial connection of segments with different types of conductivity (Matzui et al., 2005). Finally, the thermopower of NCM containing multi-wall CNTs and a low amount of amorphous carbon was shown to be determined by two components, namely, the diffusion and phonon thermopowers. The phonon thermopower dominates at very low temperatures, while the diffusion contribution prevails at higher temperatures.

\section{References}

Hishiyama, Y., Kaburagi, Y., \& Ono, A. (1978). Variable-range hopping condition and negative magnetoresistance of disordered carbons at low temperature. Carbon, 17, 265-276. http://dx.doi.org/10.1016/0008-6223(79)90086-1

Kaiser, B., Flanagan, G. U., Stewart, D. M., \& Beagleholel, D. (2001). Heterogeneous model for conduction in $\begin{array}{llllll}\text { conducting polymers and carbon nanotubes. Synth. } & \text { 117, }\end{array}$ http://dx.doi.org/10.1016/S0379-6779(00)00540-3 
Kang, J. L., Li, J. J., Du, X. W., Shi, C. S., Zhao, N. Q., Cui, L., \& Nash, P. (2008). Synthesis and growth mechanism of metal filled carbon nanostructures by CVD using Ni/Y catalyst supported on copper. Journal of Alloys and Compounds, 456, 290-296. http://dx.doi.org/10.1016/j.jallcom.2007.02.078

Klein, C. (1964). STB-model and transport properties of pyrolitic graphites. J. Appl. Phys., 35, 2947-2957. http://dx.doi.org/10.1063/1.1713135

Kotosonov, S. (1979). Temperature dependence of Fermi level and charge carriers concentration in carbon materials with turbostratyc structure. Construct. Mater. Carbon, 14, 55. (in Russian)

Liu, B., Sundqvist, B., \& Andersson, O. (2001). Electric resistance of single-walled carbon nanotubes under hydrostatic pressure. Sol. State Commun., 118, 31-36. http://dx.doi.org/10.1016/S0038-1098(01)00034-5

Matzui, L. Yu., Ovsienko, I. V., Len, T. A., Prylutskyy, Yu., I., \& Scharff, P. (2005). Transport properties of composites with carbon nanotube-based composites. Fullerenes, Nanotubes and Carbon Nanostructures, 13, 259-265.

Ovchinnikov, A. A., \& Atrazchev, V. V. (1998). Magnetic susceptibility of multiwall carbon nanotubes. Phys. Sol. St., 40, 1950-1954.

Ovsienko, I., Matzui, L., \& Vovchenko, L. (2001). Mechanisms of thermopower in carbon materials with different crystal structures. Low Temp. Phys., 27, 68-72

Ovsienko, I. V., Len, T. A., Matzui, L. Yu., Golub, O. A., Prylutskyy, Yu. I., \& Eklund, P. (2006). The effect of thermal and chemical treatment on the structural and phase composition of nanocarbon materials. Mater. Sci. Engineer. C, 26, 1180-1184.

Ovsienko, V., Len, T. A., Matzui, L. Yu., Prylutskyy, Yu. I., Ritter, U., Scharff, P., Le Normand, F., \& Eklund, P. (2007). Resistance of nanocarbon material containing nanotubes. Mol. Cryst. Liq. Cryst., 468, 289-297.

Ovsienko, I. V., Matzuy, L. Yu., Zakharenko, M. I., Len, T. A., Strzhemechny, Y. M., \& Eklund, P. (2008). Magnetometric studies of catalyst refuses in nanocarbon materials. Nanoscale Res. Lett., 3, 60-64. http://dx.doi.org/10.1007/s11671-007-9115-z

Piraux, L., Kinany-Alaoui, M., Issi, J. P., \& Perignon, A. (1988). Thermoelectric power and thermal coductivity of first-stage graphite-acceptor intercalation compounds. Phys. Rew. B, 38, 4329-4332. http://link.aps.org/doi/10.1103/PhysRevB.38.4329

Prilutskiy, O., Katz, E. A., Shames, A. I., Mogilevsky, D., Mogilko, E., Prilutskiy, E., \& Dub, S. N. (2005). Synthesis of carbon nanomaterials by a catalytic disproportionation of carbon monoxide. Fullerenes, $\begin{array}{llll}\text { Nanotubes } \quad \text { and } & \text { Narbon }\end{array}$ http://www.tandfonline.com/doi/full/10.1081/FST-200039204\#tabModule

Shiraishi, M., \& Ata, M. (2002). Conduction mechanism in single wall nanotubes. Synth. Met., 128, 235-239.

Song, S. N., Wang, X. K., Chang, R. P. H., \& Ketterson, J. B. (1994). Electronic properties of graphite nanotubes from galvanomagnetic effect. Phys. Rev. Lett., 72, 697-700.

Vovchenko, L. L., Matzui, L. Yu., \& Ovsienko, I. V. (2000). Thermopower of pregraphitic carbons. Mol. Cryst. Liq. Cryst., 340, 361-366. 